\title{
The Need for Focused Literacy Training in the Medical School Curriculum: A Cross-Sectional Study of Undergraduate Students
}

\author{
Joyce Kling, ${ }^{1}$ Sanne Larsen, ${ }^{1}$ and Simon Francis Thomsen ${ }^{2,3}$ \\ ${ }^{1}$ Centre for Internationalisation and Parallel Language Use, Faculty of Humanities, University of Copenhagen, Copenhagen, Denmark \\ ${ }^{2}$ Department of Biomedical Sciences, Faculty of Health and Medical Sciences, University of Copenhagen, Copenhagen, Denmark \\ ${ }^{3}$ Department of Dermatology, Bispebjerg Hospital, Copenhagen, Denmark
}

Correspondence should be addressed to Joyce Kling; joyce@hum.ku.dk

Received 4 June 2017; Revised 1 August 2017; Accepted 14 August 2017; Published 19 December 2017

Academic Editor: P. Ravi Shankar

Copyright (c) 2017 Joyce Kling et al. This is an open access article distributed under the Creative Commons Attribution License, which permits unrestricted use, distribution, and reproduction in any medium, provided the original work is properly cited.

\begin{abstract}
Introduction. Medical education programs have increasingly included compulsory research skills components but rarely include explicit academic literacy instruction for medical research. This article presents results from a project that developed methods of bridging the gap between textbook literacy and scientific literacy in a setting where English coexists with the local language. Methods. A paper-based, revised version of a validated self-report instrument (32 questions) designed to assess readers' metacognitive awareness and perceived use of academic reading strategies was used to collect information about medical students' awareness of reading strategies in English for academic purposes. Results. Students reported a total overall average of 3.25 (scale 1-5) for reading strategy use, falling within a medium range for usage. They reported using problem-solving reading strategies to the greatest extent (3.76), with global reading strategies (3.29) being second, and support reading strategies (2.85) to the least extent. Based on the data, a curricular intervention was designed to support critical reading of empirical literature in English. Conclusion. The results from this study suggest the need for inclusion of focused training on academic and scientific literacy, in particular, strategy instruction in relation to foreign language reading comprehension skills in medical school curricula.
\end{abstract}

\section{Background}

The past decade has witnessed greater focus on enticing early career medical professionals into an appreciation of medical research and training them to be competent consumers of a complex field of research with many vested interests. This has increasingly led to the addition of compulsory research skills components in medical curricula $[1,2]$ with recommendations for inclusion at the undergraduate level [3], with positive student response [2, 4-6]. One example of this type of initiative is in the medical program at the University of Copenhagen (UCPH), where the majority of students engage in research during their medical training, for example, as part of their bachelor or master's projects or as part of an extracurricular undergraduate research year. At present, more than $10 \%$ of all recent graduates have completed an undergraduate research year at the Faculty of Health and Medical Sciences (SUND). To support students' development of research skills, the medical school at SUND has incorporated medical research methodology training at all levels of the program, namely, in a research methodology course at the bachelor level, a research semester/month at the master's level, an extracurricular pregraduate research year, a designated "Career Day," and the support of student run academic associations.

Until recently, however, the program did not include a focus on the development of specific academic literacy training for medical research, particularly for critical reading in English for nonnative English speaking (NNS) medical students. While these students typically present themselves as academically strong and independent learners [7, 8], NNS students have often been left to manage on their own in relation to English-medium instruction (EMI) in nonAnglophone settings [9]. The undergraduate medical curriculum at $\mathrm{UCPH}$ consists of written input almost entirely presented in the form of textbooks (the majority in Danish), whereas original scientific articles published in English 
language, peer-reviewed journals are used to a very limited extent. This article presents results from a research-supported collaborative project between UCPH's Centre for Internationalisation and Parallel Language Use (CIP) and SUND. Language training experts from CIP and medical research experts from SUND together developed ways of bridging the gap between textbook literacy and scientific literacy in a parallel language setting, where Danish and English coexist for teaching and learning. Based on the project, we discuss the value of coupling instruction on critical appraisal of research literature with awareness-raising of reading strategies and explicit language instruction based on genre theory.

$\mathrm{UCPH}$ enrolls approximately 550 undergraduates in Medical Sciences each year. The BSc requires 180 ECTS over the course of six semesters. The degree consists of 20 courses covering topics in biomedicine, cellular and molecular medicine, neuroscience and pharmacology, international health, immunology and microbiology, and public health. All of these courses are taught in Danish using mainly Danish language textbooks. During the last semester, however, students must submit a bachelor project or thesis which requires extensive reading and analysis of material published in English.

Recognizing the need to support students' thesis writing process in their final semester, SUND introduced a course in autumn 2014 on medical research methodology including the nature of research in the form of hypothesis generation, hypothesis testing and dissemination of results, literature search, critical appraisal of scientific literatures, fundamentals of biostatistics, and structure of scientific articles. This research methodology course provides students with an introduction to the basic concepts of medical research, the tools to guide them through their bachelor project, and a foundation to help them make the transition to graduate studies, should they so choose.

Unlike the other required courses for the BSc, the texts in this course are from internationally recognized journals published in English. These texts differ greatly in style and genre from the predominantly Danish medical textbooks that dominate the majority of the other courses in the bachelor degree program. The genre of the journal article can be quite challenging for undergraduate students to navigate, particularly in their second language (L2). In the transition to scientific research literature, the medical students are confronted with texts that are not pedagogically prepared for classroom use. Because of this, the research methodology instructors had concerns regarding the students' abilities to critically appraise original research in English due to the language.

Although students enter this degree program with strong overall academic skills, the majority stem from a homogeneous upper secondary school environment where their English language literacy training mainly focuses on reading, speaking, and writing about fiction [16]. The curricula in the initial period of the medical degree program requires students to (a) read for basic comprehension, drawing on skills of recall and summarization, and (b) read to learn, connecting new information with background knowledge. However, interacting with empirical medical research requires students to read to integrate: "readers must integrate the rhetorical and contextual information found across the texts and generate their own representation of this interrelationship (p. 176)" [17]. With limited knowledge of this specific genre, many students struggle to critically analyze the arguments put forward in empirical studies. As less-skilled readers, these students tend to be limited in their metacognitive knowledge about reading and do relatively little monitoring of their own memory, comprehension, and other cognitive tasks. Instead, they tend to focus on reading as a decoding process and not a meaning-getting process. In comparison, with focused training, more skilled readers can engage in deliberate activities that require self-monitoring and strategic planning [10].

To address these concerns, it was important to (a) determine the students' awareness of their reading strategies and (b) introduce the students to the text and the language functions of medical research articles (genre). Thus, a two-stage intervention was implemented. Stage 1 consisted of data collection in relation to students' metacognitive reading strategies. Stage 2 took the form of an adjunct pilot workshop that was developed as a component to the standard course on medical research. The workshop supported the students in relation to the critical reading of the empirical scientific literature in English. These two stages are outlined in Table 1.

\section{Methods}

To collect information about the students' self-perception and awareness of reading strategies in English for academic purposes (EAP), we utilized a revised version of the Metacognitive Awareness of Reading Strategies Inventory (MARSI) [10]. The MARSI is a self-report instrument designed to assess adolescent and adult readers' metacognitive awareness and perceived use of reading strategies while reading academic or school-related materials. The MARSI is comprised of 30 strategies:

(i) 13 Global Reading Strategies (GRS: generalized, intentional reading strategies aimed at setting the stage for the reading act).

(ii) 8 Problem-Solving Strategies (PSS: localized, focused problem-solving or repair strategies used when problems develop in understanding textual information when the text becomes difficult to read).

(iii) 9 Support Reading Strategies (SRS: use of outside reference materials aimed at sustaining responses to reading).

Our revised MARSI included two additional SRS drawn from Mokhtari and Sheorey's [15] Survey of Reading Strategies (SORS), an adaptation of the MARSI for an English as a second language (ESL) population. On the MARSI, students rate their perception of use in response to the listed statements about what people do when they read academic materials like textbooks, articles, and so on in English on a 5-point Likerttype scale from 1 to 5 , with 1 indicating "I never or almost never do this" and 5 indicating "I always or almost always do this." In addition to the MARSI survey, students were asked to 
TABLE 1: Two-stage curricular intervention.

\begin{tabular}{|c|c|c|c|}
\hline & Curricular intervention & Purpose & Content \\
\hline Stage 1 & $\begin{array}{l}\text { Administration of the Metacognitive } \\
\text { Awareness of Reading Strategies } \\
\text { Inventory (MARSI), a self-report } \\
\text { instrument designed to assess adolescent } \\
\text { and adult readers' metacognitive } \\
\text { awareness and perceived use of reading } \\
\text { strategies while reading academic or } \\
\text { school-related materials [10] }\end{array}$ & $\begin{array}{l}\text { To determine the students' } \\
\text { self-perception of reading strategies in } \\
\text { English for academic purposes } \\
\text { To raise the students' awareness of } \\
\text { reading strategies in English for academic } \\
\text { purposes }\end{array}$ & $\begin{array}{l}32 \text { questions related to } \\
\text { (i) Global Reading Strategies } \\
\text { (ii) Problem Solving Strategies } \\
\text { (iii) Support Reading Strategies } \\
\text { Plus } \\
\text { (i) Biodata: age and gender } \\
\text { (ii) Foreign language proficiency }\end{array}$ \\
\hline Stage 2 & $\begin{array}{l}\text { Implementation of an adjunct workshop } \\
\text { conducted cooperatively by a medical } \\
\text { professor and an academic language } \\
\text { consultant } \\
\text { Two interactive sessions integrated into } \\
\text { the regularly scheduled course }\end{array}$ & $\begin{array}{l}\text { To develop critical reading strategies in } \\
\text { relation to domain specific } \\
\text { argumentation: } \\
\text { (i) Stylistic and rhetorical English } \\
\text { language usage in empirical articles in } \\
\text { scientific publications } \\
\text { (ii) Linguistic characteristics used to } \\
\text { build an argument in empirical articles in } \\
\text { scientific publications }\end{array}$ & $\begin{array}{l}\text { Reading strategies } \\
\text { (i) Global Reading Strategies } \\
\text { (ii) Problem Solving Strategies } \\
\text { (iii) Support Reading Strategies } \\
\text { Genre analysis } \\
\text { (i) Rhetorical moves (IMRAD) [11-13] } \\
\text { (ii) Hedging and boosting devices [14] }\end{array}$ \\
\hline
\end{tabular}

provide biodata and self-assessment of their foreign language proficiency.

137 third-year undergraduate medical students (67\% female, $33 \%$ male) with an average age of 22.0 years enrolled in the medical research methodology course in spring 2016 responded to the paper-based survey (83\% reported Danish as their first language). Each question was scored on a Likerttype scale from 1 to 5 with, 1 indicating low and 5 indicating high proficiency. Overall and domain-specific mean values were calculated for GRS, PSS, and SRS. Differences in scores were explored for gender, age, self-rated language proficiency, and first language. Reliability analysis and principal components analysis were used to examine the internal consistency and correlational structure of the data and to enable comparison with the original validation reported by Mokhtari and Reichard [10]. A three-component solution retaining components (1) a global reading strategies component, (2) a problem-solving reading strategies component, and (3) a support reading strategies component with varimax rotation with Kaiser normalization was reported.

\section{Results}

Table 2 lists the results for the 137 respondents. The medical students reported a total overall average of 3.25 for strategy use, falling within a medium range for usage. They reported using PSS to the greatest extent (3.76), with GRS (3.29) being second, and SRS (2.85) to the least extent. These all fall in the medium range for usage. The top four strategies that the students report using when reading academic materials like textbooks, articles, and so on in English were one global reading strategy (strategy number (17), I use tables, figures, and pictures in text to increase my understanding) and three problem-solving reading strategies (strategies number (13), $I$ adjust my reading speed according to what I'm reading; (11), I try to get back on track when I lose concentration; and (27), When text becomes difficult, I re-read to increase my understanding), whereas the four bottom strategies were three support reading strategies and one global reading strategy.

Cronbach's alpha values were 0.80 for GRS, 0.65 for PSS, and 0.54 for SRS, whereas the reliability for the total sample was 0.83 , suggesting a high overall reliability but a lower internal consistency for the three specific reading strategy components compared with the original validation of the MARSI tool [10], where the same values for overall consistency as well as the three reading strategies components ranged 0.89-0.93. Our principal components analysis yielded three components with high eigenvalues (above 2.0), GRS, 6.22; PSS, 2.43; and SRS, 2.34, whereas another eight components had eigenvalues in the range of 1.04 to 1.74 (Figure 1). Most of the 32 individual reading strategies loaded strongest on the reading strategies component, that is, the global reading strategies component, the problem-solving reading strategies component, or the support reading strategies component, respectively, originally allocated to that individual reading strategy in the validation reported by Mokhtari and Reichard [10] (Table 2). However, deviance from this was particularly seen for strategies number (4), I take an overall view of the text to see what it is about before reading it; (8), I read slowly but carefully to be sure I understand what I'm reading; (13), I adjust my reading speed according to what I'm reading; (16), When text becomes difficult, I pay closer attention to what I'm reading; (24), I go back and forth in the text to find relationships among ideas in it; (26), I try to guess what the material is about when I read; and (27), When text becomes difficult, I re-read to increase my understanding, which all loaded markedly stronger on another reading strategies component than originally. Particularly, most deviance was observed from the problem-solving reading strategies component (4 items: (8), (13), (16), and (27)), whereas least deviance was observed from the global reading strategies component (2 items: (4) and (26)) and the support reading strategies component (1 item: (24)), respectively. This suggests that the applicability, reliability, and interpretation of the MARSI results, particularly the problem-solving reading 


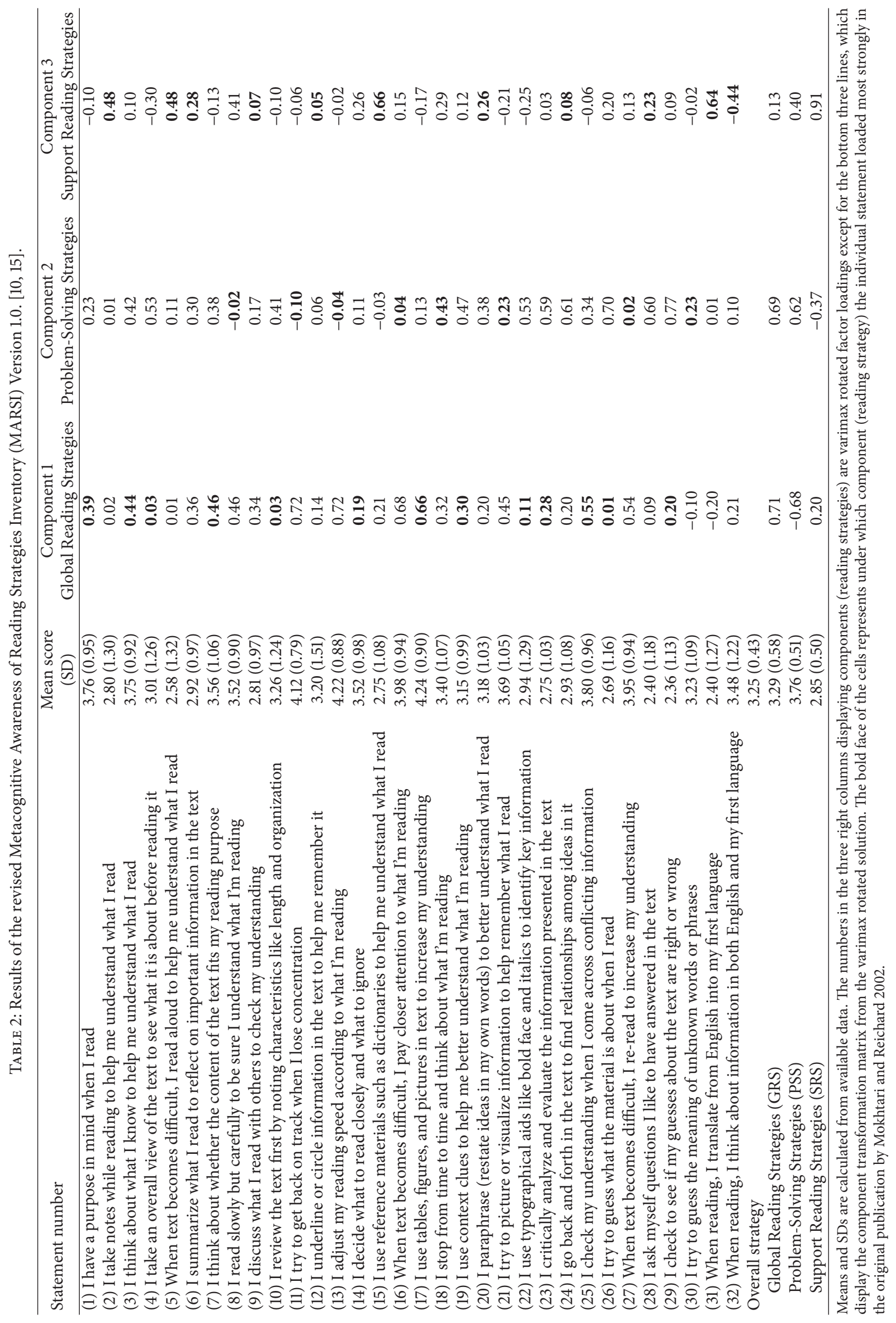




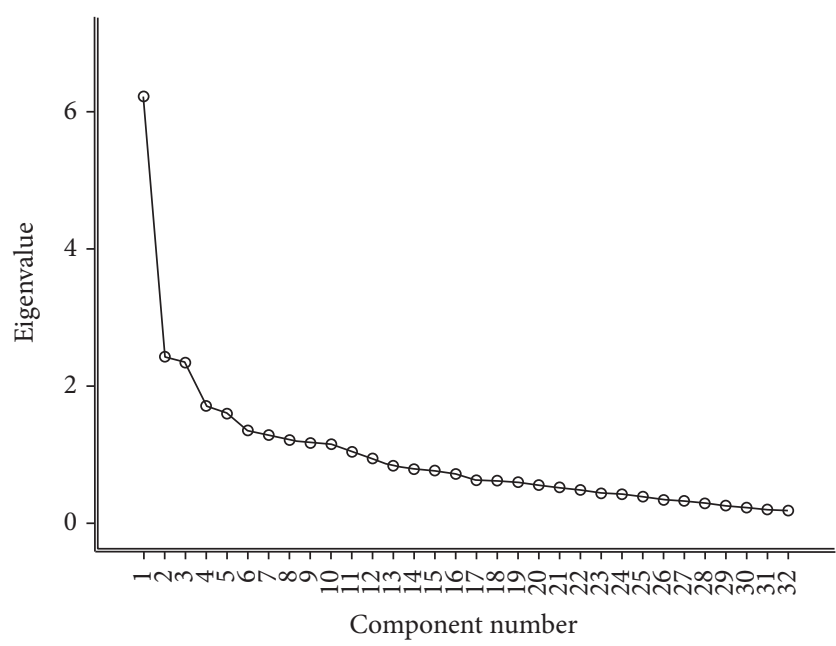

FIGURE 1: Scree plot of the results of a principal components analysis of the 32-item MARSI questionnaire among medical undergraduate students.

TABLE 3: Biographical data and language proficiency.

\begin{tabular}{ll}
\hline Gender (number, \%) & \\
$\quad$ Female & $86(67.2)$ \\
$\quad$ Male & $42(32.8)$ \\
Age, years & $22.0(1.5)$ \\
First language, Danish (number, \%) & $105(83.3)$ \\
Self-rated language proficiency & \\
$\quad$ Speaking English & $3.78(0.79)$ \\
Understanding spoken English & $4.42(0.68)$ \\
Writing academic papers & $2.98(0.99)$ \\
$\quad$ Reading academic texts & $3.60(0.86)$ \\
\hline
\end{tabular}

Numbers are calculated from available data.

strategies, depend on the educational setting, which in this case included medical undergraduate students and not high school students. Further, we used a 32-item checklist compared with the original 30 item checklist, which might also have introduced some inconsistency. Nonetheless, our analysis did not aim to explore a whole new factor structure of the observed data but rather to employ the proposed three-factor structure suggested in the original validation of the model by exploratory factor analysis. Particularly, the original publication recommended evaluating the scree plot, the eigenvalues, and the interpretability of factors in tandem to decide the number of factors to retain [10], which is in good alignment with our model.

The students' self-reported language proficiency on a 5point Likert-type scale averaged 3.78 for speaking, 4.42 for understanding spoken language, 2.98 for writing, and 3.60 for reading academic papers (Table 3 ).

Age was not statistically significantly associated with overall or domain-specific strategy use, and there were no statistically significant differences between female and male students for overall strategy use (3.26 versus 3.24), PSS (3.79 versus 3.74 ), GSS (3.39 versus 3.22 ), or SRS (2.91 versus
2.74). Similarly, first language and self-reported language proficiency was not statistically significantly associated with overall or domain-specific strategy use when adjusting for multiple testing.

\section{Discussion and Conclusion}

This paper has considered support for developing medical students' research skills through the lens of L2 reading strategy awareness and development. This kind of support for disciplinary learning is often ignored in medical schools in both Anglophone and non-Anglophone contexts. However, universities cannot assume that students come to higher education with adequate skills for advanced level, disciplinary critical reading, especially in a foreign language [18], or that they develop these skills without systematic support. In academic settings where students are reading and discussing material in their foreign/second language, students must cope with the challenge of both language and content. One way of addressing this challenge is by building English languagefocused elements into existing courses in a program, aligning them with the disciplinary learning goals of these courses.

Using a validated instrument such as the MARSI to reinforce students' awareness of strategies for reading, and more specifically reading in a foreign language, provided a needs assessment as a first step toward integrating English language training into the medical research course. The medical research course includes a standard introductory session where the basic tenets of scientific research are introduced. At the end of this session, the students receive a list of questions that they should be asking to critically assess research literature. However, the ability to engage with these questions may rely on reading strategies that are not part of the students' strategic repertoire or that they have used only sporadically to engage with other genres, such as textbooks. As noted in the MARSI data, the students do not report using global reading strategies to a high extent. Instead they report using problem-solving strategies to the greatest extent. This would allow them to get through the text, to get the reading done, but it might not be sufficient to critically appraise the research articles.

With baseline knowledge of the students' approaches to reading in EAP from the MARSI, we were able to build an adjunct language-focused component that aligned with the goals of the medical research course. Drawing on genre approaches to reading instruction, the intervention took the students through both the structure ("rhetorical moves") and the linguistic characteristics (e.g., word choice, hedging, boosting, and use of modals) of empirical research published in English within their field. These instructional foci have been shown to capture central differences between textbooks and research articles and to provide L2 students with concrete points to aid in the reading process [11]. The assumption was that once the students had identified these aspects, they could better form an opinion about the domain-specific argumentation, for example, the strength and validity of a study. In other words, at this stage, they could go back and use the information and questions provided about research more efficiently. A follow-up study is needed to assess the effect 
of the language-focused intervention [12], both in terms of supporting students' ability to engage with research critically in the medical research course and their ability to use appropriate reading strategies and genre knowledge for writing their BA projects, which was the motivation for introducing the medical research course in the curriculum. Numerous factors have a bearing on medical students' ability and motivation to engage with research, but it starts with students having the reading skills to engage with the genre(s) and language(s) of medicine when they encounter them in the curriculum.

\section{Conflicts of Interest}

The authors declare that they have no conflicts of interest.

\section{References}

[1] D. P. Wickramasinghe, C. S. Perera, S. Senarathna, and D. N. Samarasekera, "Patterns and trends of medical student research," BMC Medical Education, vol. 13, no. 1, article no. 175, 2013.

[2] D. T. Laskowitz, R. P. Drucker, J. Parsonnet, P. C. Cross, and N. Gesundheit, "Engaging students in dedicated research and scholarship during medical school: The long-term experiences at Duke and Stanford," Academic Medicine: Journal of the Association of American Medical Colleges, vol. 85, no. 3, pp. 419$428,2010$.

[3] A. Lawson McLean, C. Saunders, P. P. Velu, J. Iredale, K. Hor, and C. D. Russell, "Twelve tips for teachers to encourage student engagement in academic medicine," Medical Teacher, vol. 35, no. 7, pp. 549-554, 2013.

[4] D. Moßhammer, M. J. Roos, A. Kronenthaler et al., "Students' performing of practical research tasks for their scientific qualification - an approach within the family practice internship in undergraduate education," GMS Z Med Ausbild, vol. 28, no. 24, 2011.

[5] R. L. Houlden, J. B. Raja, C. P. Collier, A. F. Clark, and J. M. Waugh, "Medical students' perceptions of an undergraduate research elective," Medical Teacher, vol. 26, no. 7, pp. 659-661, 2004.

[6] D. Hren, I. K. Lukić, A. Marušić et al., "Teaching research methodology in medical schools: students' attitudes towards and knowledge about science," Medical Education, vol. 38, no. 1, pp. 81-86, 2004.

[7] K. W. Eva, "Reading means more than deciphering the words on the page," Medical Education, vol. 44, no. 4, pp. 330-332, 2010.

[8] D. S. McNamara, "Strategies to read and learn: Overcoming learning by consumption," Medical Education, vol. 44, no. 4, pp. 340-346, 2010.

[9] T. Hauge, "Language excellent - a necessary skill? University lecturers' dilemmas in teaching content courses in English as an international language," in Policies, principles, practices: new directions in foreign language education in the era of educational globalization, R. Cancino, L. Dam, and K. Jæger, Eds., Newcastle upon Tyne, pp. 161-187, Cambridge Scholars Publishing, 2011.

[10] K. Mokhtari and C. A. Reichard, "Assessing students' Metacognitive Awareness of Reading Strategies," Journal of Educational Psychology, vol. 94, no. 2, pp. 249-259, 2002.
[11] S. Hyon, "Long-term effects of genre-based instruction: A follow-up study of an EAP reading course," English for Specific Purposes, vol. 20, no. 1, pp. 417-438, 2001.

[12] D. Kirkpatrick, "Revisiting Kirkpatrick's four-level-model," Training \& Development, vol. 1, pp. 54-57, 1996.

[13] K. N. Nwogu, "The medical research paper: Structure and functions," English for Specific Purposes, vol. 16, no. 2, pp. 119138, 1997.

[14] J. Skelton, "Analysis of the structure of original research papers: An aid to writing original papers for publication," British Journal of General Practice, vol. 44, pp. 455-459, 1994.

[15] K. Mokhtari and R. Sheorey, "Measuring ESL students awareness of reading strategies," Journal of Developmental Education, p. 25, 2002.

[16] M. Mondahl, B. Henriksen, M. Skovgaard Andersen et al., "A study of foreign language instruction in upper secondary school," http://openarchive.cbs.dk/bitstream/handle/10398/8320/ Sprogkernen.pdf?sequence $=1$.

[17] L. Trites and M. McGroarty, "Reading to learn and reading to integrate: New tasks for reading comprehension tests?" Language Testing, vol. 22, no. 2, pp. 174-210, 2005.

[18] G. O. Hellekjær, "Academic English reading proficiency at the university level: a Norwegian case study," Read Foreign Lang, vol. 21, no. 2, 2009. 


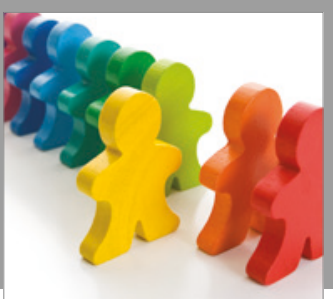

Autism

Research and Treatment
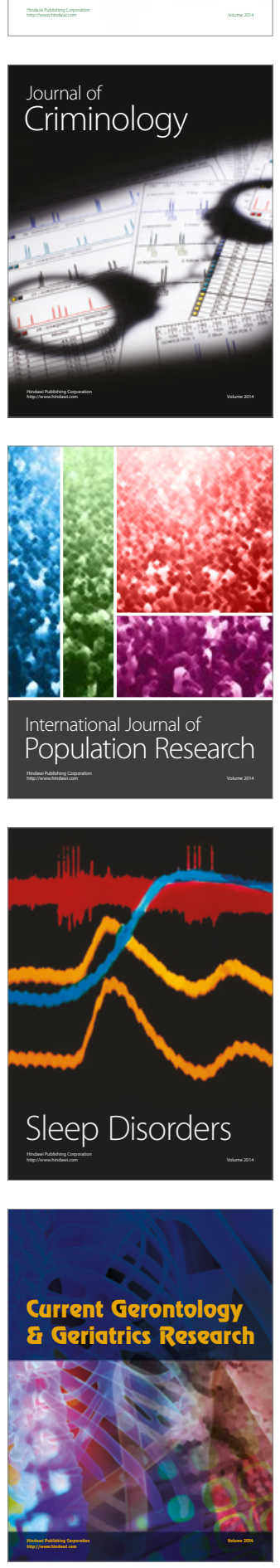

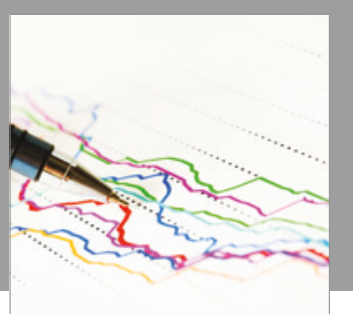

Economics

Research International

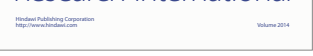

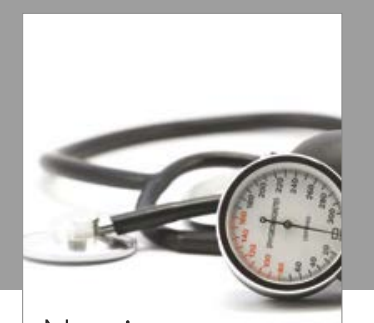

Nursing

Research and Practice

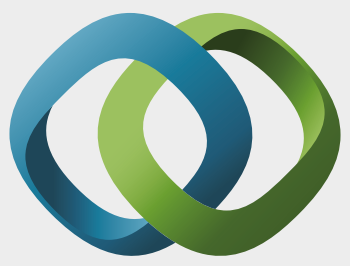

\section{Hindawi}

Submit your manuscripts at

https://www.hindawi.com
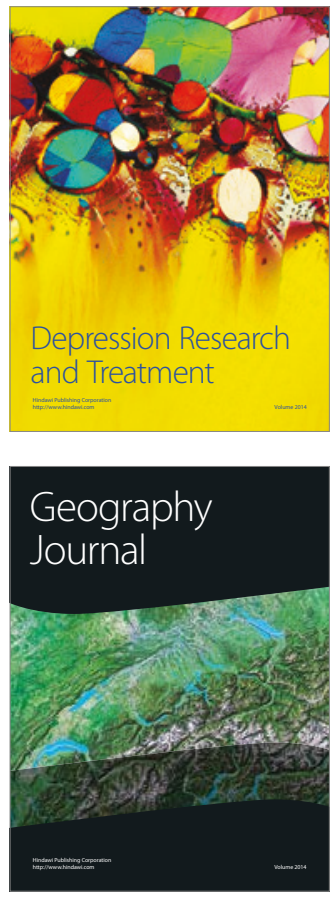
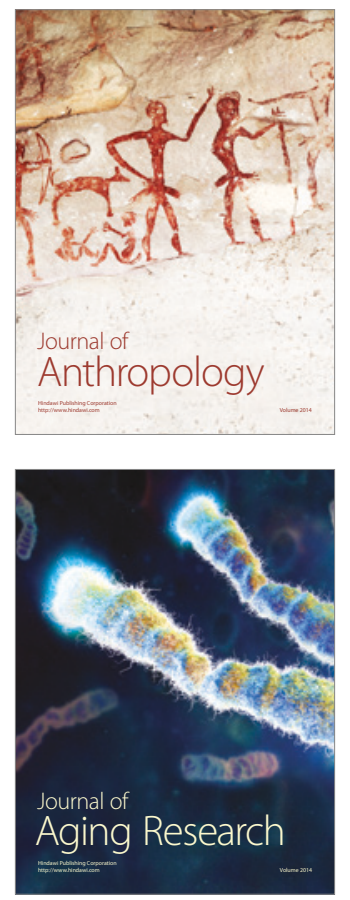
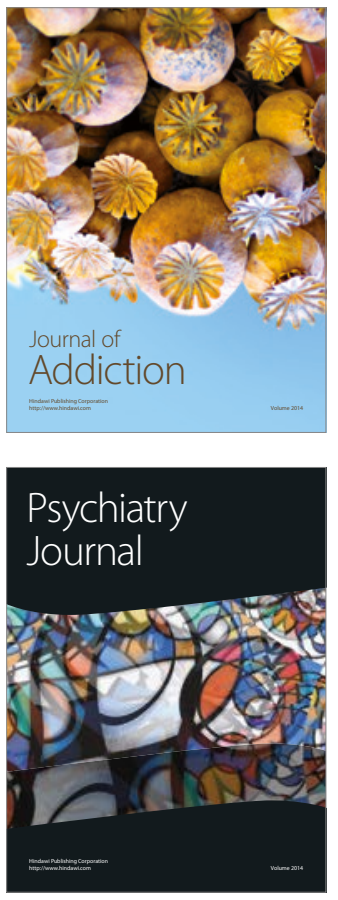

Child Development

Research

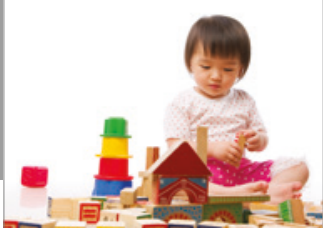

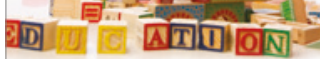
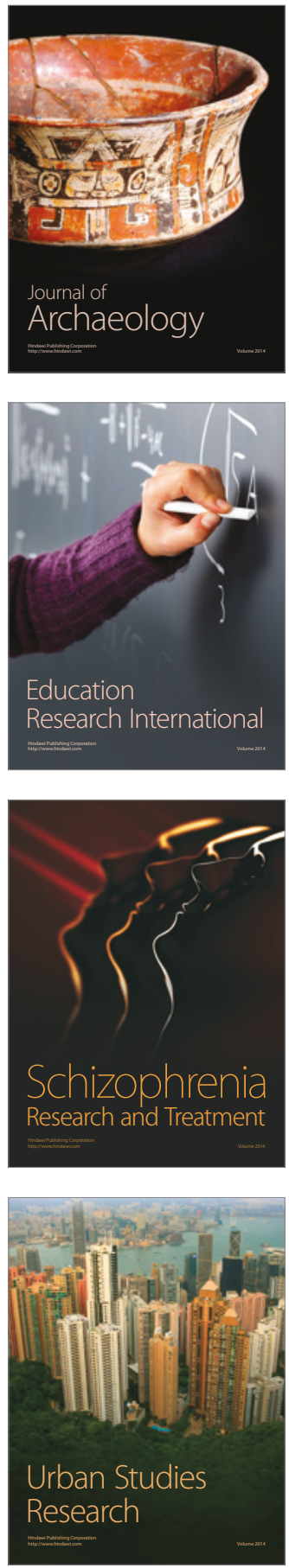